\title{
Earthquake-related Electric Field Changes Observed in the lonosphere and Ground
}

\author{
Yingying FAN, Xuebin DU, Zhanghui AN, Jun LIU, Dacheng TAN, \\ and Junying CHEN
}

Lanzhou Base of Institute of Earthquake Prediction, China Earthquake Administration, Lanzhou City, China; e-mail: fyy416@163.com

Lanzhou Institute of Seismology, China Earthquake Administration, Lanzhou City, China

\begin{abstract}
The changes of the ionospheric electric field before and after four huge earthquakes, which include the Ms 8.7 earthquake of 2004 and the Ms 8.5 earthquake of 2005 in Sumatra of Indonesia, the Ms 8.0 Wenchuan earthquake of 2008 in China, the Ms 8.8 earthquake of 2010 in Chile, and their strong aftershocks are studied in this paper. The significant results revealed that the power spectral density of low-frequency electric field below $20 \mathrm{~Hz}$ in the ionosphere, a kind of electromagnetic radiation phenomena, increased abnormally before and after the earthquakes and partially corresponded to the increased power spectral density of the low-frequency geoelectric field in time. This research preliminarily indicates that the low-frequency electromagnetic radiation during the imminent stages before such earthquakes could be detected by the observation of the ionospheric electric field. However, the spatial, temporal, and intensive complexities of the electric field anomalies in the ionosphere before earthquakes have come in sight also.
\end{abstract}

Key words: ionosphere, satellite, electric field, earthquake, changes. 


\section{INTRODUCTION}

Recently, more and more attention is paid internationally to the application of electromagnetic observations in the earthquake (EQ) and volcano monitoring. Since the 1950s, the former Soviet Union, Japan, the United States, China, and other countries have carried out the ground electromagnetic observations and reported the electromagnetic precursory phenomena in the time and frequency domains before earthquakes (Varotsos et al. 1986, Varotsos and Lazaridou 1991, Michel and Zlotnicki 1998, Zlotnicki et al. 2001, Uyeda et al. 2002, Ma et al. 2004, Telesca et al. 2005, 2008, 2009, Enomoto et al. 2006, Telesca and Hattori 2007, Nagamoto et al. 2008, Tang et al. 2008, Fan et al. 2010, Liu et al. 2011). Furthermore, a lot of loading experiments and theoretical studies have been made on the mechanism of generation and propagation of the electromagnetic signals (Ogawa et al. 1985, Cress et al. 1987, Yamada et al. 1989, Enomoto and Hashimoto 1992, Varotsos et al. 1998, Huang and Ikeya 1998, Adler et al. 1999, Ishido and Pritchett 1999, Huang 2005). However, the long-term observation also reveals that the earthquake-related precursory anomalies recorded by ground stations displayed extremely complicated spatial, temporal, and intense distributions. For example, no anomalies appeared evenly in/near the epicentral areas of some strong or huge EQs, anomalies have not been repeatedly recorded before the equivalent-magnitude EQs, and even there were a lot of anomalies without any earthquake (no earthquake occurred near the station at which an electromagnetic anomaly has been recorded), as reported by $\mathrm{Du}$ (2011). These complicated phenomena cause great difficulties in identifying any earthquake-related information and determining an EQ forecasting statement.

Since the 1980s, the space-to-ground electromagnetic observation with the purpose of EQ monitoring and prediction has been carried out internationally (Parrot 1995, 2006a, b), and the electromagnetic phenomena in the ionosphere associated with EQs were reported (Liu et al. 2003, Pulinets et al. 2005, Zhang et al. 2012). This will set up a three-dimension- al electromagnetic observation system by integrating with the ground electromagnetic observations, which will perhaps help to overcome the afore- mentioned complicated problems of earthquake-related electromagnetic precursory anomalies. In 2004, France launched a special satellite, the Demeter (detection of electro-magnetic emissions transmitted from EQ regions), for the EQ electromagnetic monitoring. During the satellite's operation, a number of great EQs, with magnitude of above $M s$ 8.0, occurred globally, and a series of EQs above Ms 7.0 happened in Chinese mainland and surrounding areas. The electromagnetic precursory anomalies in the ionosphere deemed to be associated with the EQs are reported (Liu et al. 2003, 2011, Pulinets et al. 
2005, Cussac et al. 2006, He et al. 2009, Fan et al. 2010, Zhang et al. 2012). This paper will study the changes of the ionospheric electric field observed by Demeter before and after the EQs, compare the ionospheric electric fields and the ground electric/magnetic field changes before the EQs occurred in Chinese mainland, and, accordingly, report some significant results obtained.

\section{DATA AND METHOD}

The satellite Demeter was launched into orbit on 29 June 2004, as a special seismic electromagnetic observation satellite, which had a circular sunsynchronous polar orbit with an inclination of $98.23^{\circ}$ and a run altitude of $710 \mathrm{~km}$ (this altitude was changed to $665 \mathrm{~km}$ in mid-December 2005; Cussac et al. 2006, Parrot 2006a, b). During its operation, a series of EQs with the magnitude of above $M s 8.0$ occurred globally, such as the Ms 8.7 EQ (2004), $M s$ 8.5 EQ (2005), the $M s$ 8.5 and 8.3 EQs (2007) in Sumatra, Indonesia, the Ms 8.0 EQ (2008) in Wenchuan, China, and the Ms 8.8 EQ (2010) in Chile as well as the Ms 7.3 EQ (2008) in Yutian, China. This paper focuses on the ionospheric electric field changes observed by the ICE (Instrument Capteur Electrique; Berthelier et al. 2006) electric field instrument before the great EQs. The ICE electric field instrument measured the three electrical field components. The Demeter satellite adopts the "survey" mode to record the electric field data with the sampling frequency of 39.0625. Considering that the earthquake-related electromagnetic field has the characteristics of "the field distribution and propagation", the observation data of the Demeter's orbits within the latitude and longitude of $20^{\circ} \times 20^{\circ}$ range above the epicenters are selected. Under normal circumstances, the satellite runs through 2 to 3 half orbits every day within the above-mentioned range, and thus the electric field observation data of a number of days before and after the EQs may constitutes the time series that consists of many half-orbits according to time order, which is referred to as "successive orbit" in this paper.

The Ms 8.0 Wenchuan EQ and the Ms 7.3 Yutian EQ happened in the eastern and western edges of the Qinghai-Tibet Plateau, respectively, with a distance of about $2000 \mathrm{~km}$ between their epicenters. The ground electric stations, with the solid non-polarized electrodes made in China, are distributed in the northern, northeastern, and eastern edges of the Qinghai-Tibet Plateau. The station measures the three components of the geoelectric field below $0.005 \mathrm{~Hz}$, along the NS, EW, and oblique directions, respectively; its electrode spacing is 90 300 $\mathrm{m}$ and they are buried below $2 \mathrm{~m}$ depth (Qian et al. 2004, Du et al. 2006). Fan et al. (2010) and Liu et al. (2011) studied the electric/magnetic response observed on the ground before the two EQs. Referring to the ground electric/magnetic changes, this paper reports a compar- 
ative research on the ionospheric electric field changes observed by the Demeter before and after the two great EQs.

Due to the high sampling rate of the Demeter's ionospheric electric field observations, it is not suitable to analyze the waveform changes of the electric field in time domain. Fan et al. (2010) and Liu et al. (2011) applied the maximum entropy method (MEM) to conduct research on the time-varying power spectral density (PSD) of the electric/magnetic field observed by the ground electromagnetic stations, approaching to the occurrence date of the Wenchuan EQ and other strong EQs in the Qinghai-Tibet Plateau and its edge. The significant information of the time-varying PSD changes corresponding to the EQs has been obtained. This paper applies the method to process the electric field observation data in the ionosphere observed by Demeter, which is conductive to the comparative analysis for the ionospheric and ground electric field changes before EQs.

\section{IONOSPHERIC ELECTRIC FIELD CHANGES BEFORE GREAT EQS}

\subsection{Ionospheric and ground electric fields before the Wenchuan Ms 8.0 EQ}

\subsubsection{Ionospheric electric field (continual orbit)}

Here, the so-called continual orbit means all orbits of Demeter over the selected area within the required time. The Wenchuan Ms 8.0 EQ, in Sichuan, China, occurred on 12 May 2008 (LZT), with its epicenter located at $\mathrm{N} 31.01^{\circ}, \mathrm{E} 103.38^{\circ}$. The observational data of the ionosperic electric field of the Demeter's orbits, the Up-orbit observation data (night orbits) in the range of $\mathrm{N} 20^{\circ}-40^{\circ}$ and $\mathrm{E} 90^{\circ}-110^{\circ}$ and in the period from 1 January to 23 June 2008 are selected.

Figure 1 shows the time-varying PSD changes of the ionospheric electric field of the three components: $X, Y$, and $Z$ (the abscissa is the date, the vertical axis is the frequency, and the color is amplitude of PSD; the date and EQs in the figure are marked in UT). The data are observed by the Demeter above the Wenchuan and analyzed through the MEM method. Three EQs with the magnitude $M s \geq 6.9$ occurred in the selected range of the Demeter's orbit from 1 January to 23 June 2008, which were the Ms 6.9 Gaize EQ in Tibet on 9 January, the Ms 7.3 Yutian EQ in Xinjiang on 20 March, and the Ms 8.0 Wenchuan EQ on 12 May, in China. It can be seen from Fig. 1a that discontinuous PSD increase of frequency $f<3 \mathrm{~Hz}$ appeared in the three components of ionosphere electric field from 8 January 2008, which disappeared around 10 February 2008. These changes corresponded to the time of occurrence of the $M s 6.9$ Gaize EQ and its strong aftershocks. From Fig. 1b, the PSD increase of $f<3 \mathrm{~Hz}$ in the $Y$-component on 19 March, correspond- 

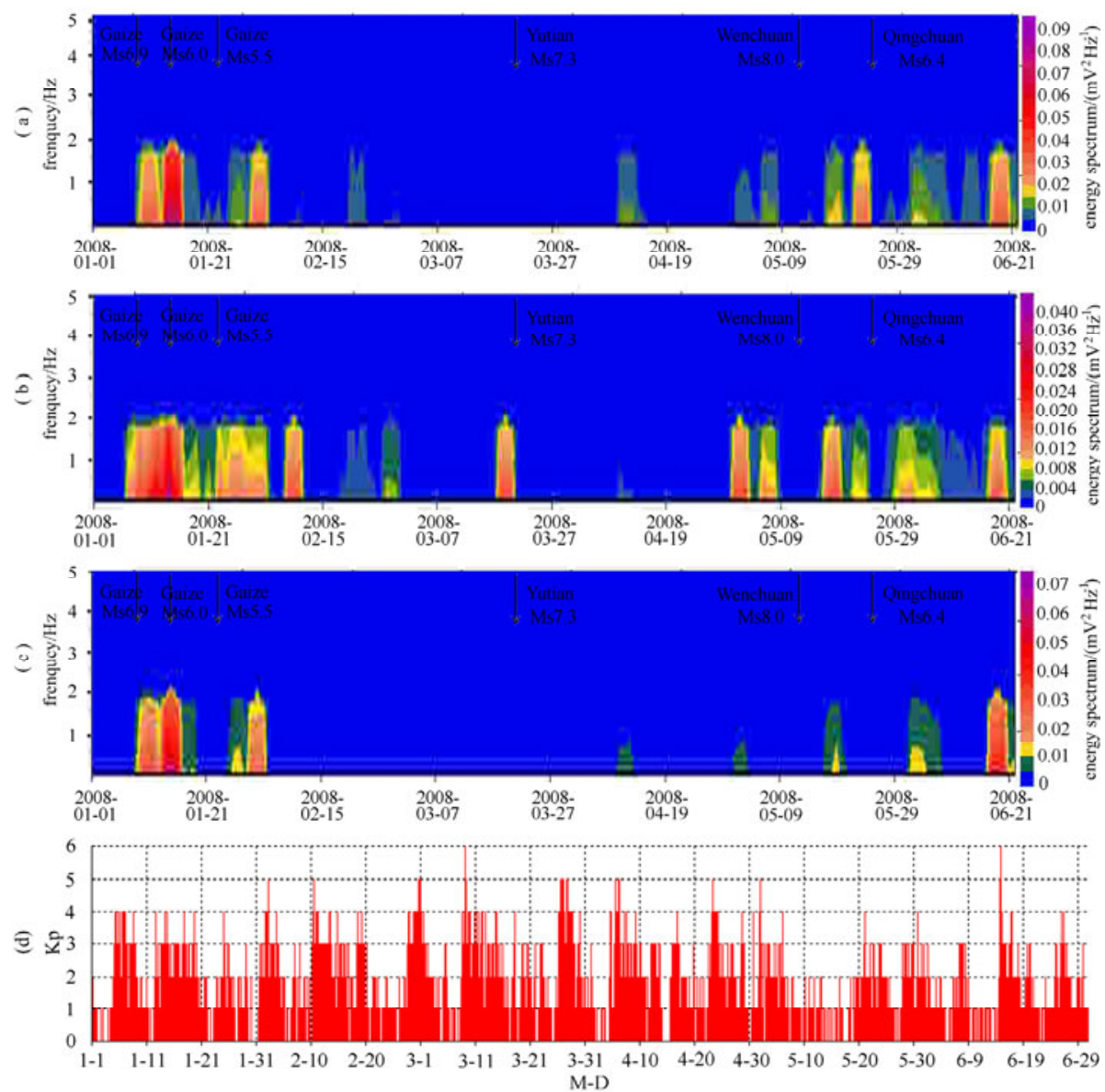

Fig. 1. Time-varing electric field PSD of Demeter's Up Orbit above Wenchuan and the $K p$ index in the corresponding period (d). Note: (a)-(c) are diagrams for $X-, Y$-, and $Z$-components.

ingly, at 22:00 of 20 March (at 6:33 of 21 March LZT), the Ms 7.3 EQ happened in Yutian county, Xinjiang. And then, since the occurrence of the Yutian EQ till the end of April, the PSD of the three electric field components has kept relatively calm (Fig. 1a-c) and during the time interval no EQ with the magnitude of $M s \geq 6$ happened globally, except for the $M s 6.1 \mathrm{EQ}$ occurred in the south Taiwan sea, China. In early May 2008, the $X$ - and $Y$ components had the PSD increase, of which the most significant one was the PSD increase of the $Y$-component, and this corresponded to the time of occurrence of the Wenchuan Ms 8.0 EQ on 12 May 2008 and its aftershocks. However, the PSD increase starting before the occurrence of the Wenchuan 
great EQ did not disappear until 23 June, and consequently a series of Ms 5.0 6.4 strong aftershocks, such as the $M s$ 6.4 Qingchuan EQ and others, occurred in the Wenchuan and its vicinity from June to July 2008. Therefore, the increased PSDs were perhaps related to these strong aftershocks.

Figure 1d shows the geomagnetic $K p$ indexes from January to June 2008. According to the analysis, the $K p$ indexes are less than 4 during the time interval of the PSD increase when approaching the date of occurrence of these EQs; however, as for the corresponding magnetic storms of $K p=6$ on 9 March and 14 June, no PSD increase appeared in the ionospheric electric field. Thus, the above-mentioned PSD increase should not be caused by magnetic activity.

From January to June 2008, in addition to the aforementioned EQs, 11 other EQs with the magnitude $M s>7.0$ occurred globally; the greater EQs corresponding to the time interval of the aforementioned PSD increase were the Sumatra $M s$ 7.7/7.6 EQs on 20 and 25 February and the Okhotsk Ms 7.6 EQ on 5 July, which were far from the selected orbit range.

\subsubsection{Comparisons between the ionospheric and ground electric field changes}

The Wenchuan Ms 8.0 EQ occurred in the eastern edge of the Qinghai-Tibet Plateau. Dozens of electromagnetic stations of the China Earthquake Administration (CEA) are distributed along the eastern, northeastern and northern edges. Two ground geo-electromagnetic stations, namely, SHN and TIA, co-established by Lanzhou Institute of Seismology (CEA-LIS) and Clermont-Ferrand Geophysical observatory of French Academy of Sciences (CNRS-OPGC) are distributed near the Tianzhu fault, China Gansu, which is located in the Northeastern Tibetan Plateau. These stations are dozens of kilometers to $1000 \mathrm{~km}$ from the epicenter of the great Wenchuan EQ. Fan et al. (2010) applied the MEM method to process the ground electric field data observed by SHN (683 km from the epicenter), TIA $(669 \mathrm{~km})$, and Chengdu station (CDU, $35 \mathrm{~km})$. Among the stations, the electrode-ranging region of stations SHN and TIA are the herdsmen's pastures, which are open and flat, without the low-frequency electromagnetic interference like plants and large electrical equipments within dozens of kilometers around. The electromagnetic environment is very favorable. Geologically, the two stations are located in the Haiyuan-Liupanshan mountain fracture zone. According to a long-term observation of EQ precursor, if the geo-electromagnetism, underground flow and crustal deformation stations are located nearby such active fracture zone, the imminent anomalies at these stations are easy to be observed immediately before EQs (Du et al. 1993, 1997, 2000). Furthermore, the rock experiments showed the medium stress in this position is sensitive to disturbed stress (Kusunose et al. 1980). 

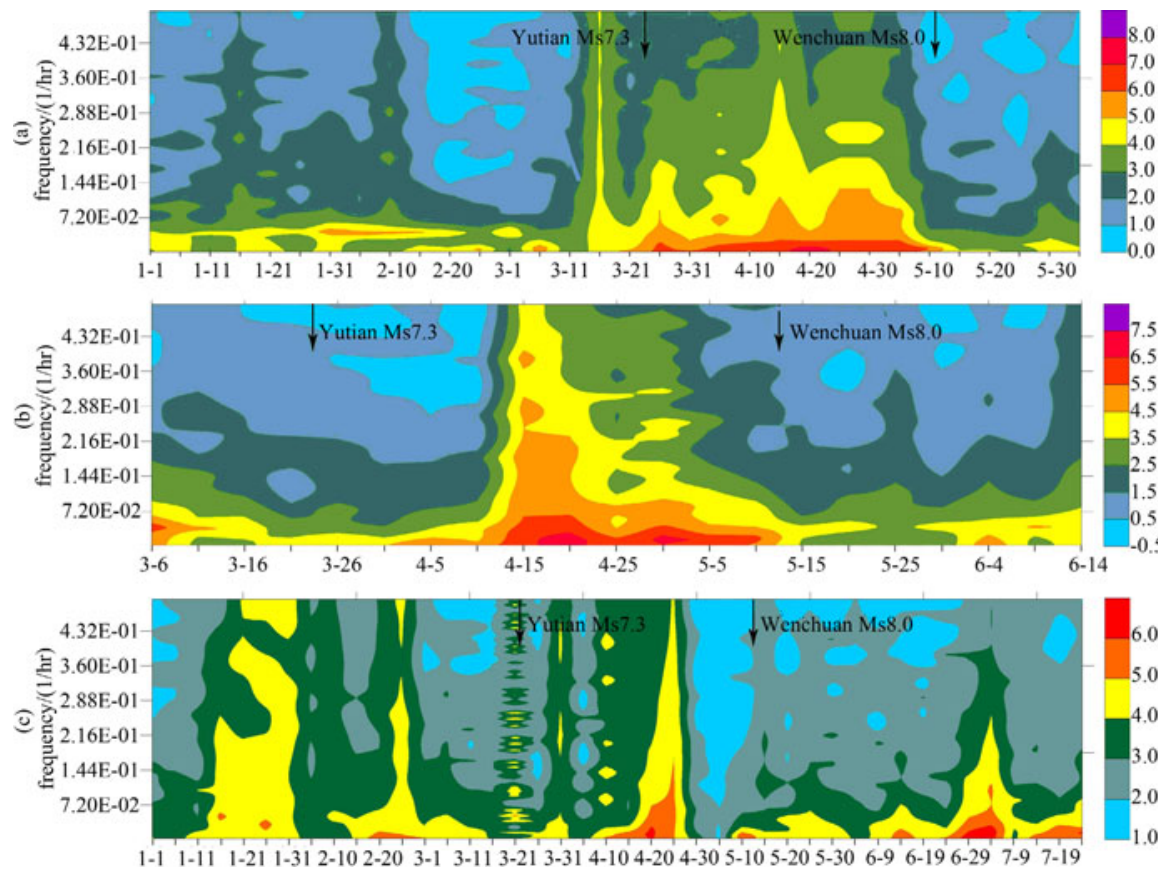

Fig. 2. MEM time-frequency diagrams for the $\mathrm{N}_{1} \mathrm{~S}_{1}$ channel of SHN (a), the $\mathrm{N}_{1} \mathrm{~S}_{1}$ channel of TIA (b), and the EW channel of CDU (c) stations. Note: Color values are $\operatorname{Lg}(\mathrm{PSD})$.

Figure 2 shows the time-varying PSD of the geoelectric field data of the $\mathrm{N}_{1} \mathrm{~S}_{1}$ channel of station SHN, the $\mathrm{N}_{1} \mathrm{~S}_{1}$ channel of station TIA, and the EW channel of station CDU (the date in the figure is in local time), which are processed by using the MEM method. In the calculation of PSD, every 5-day continuous observation data constitutes a time series and is used to calculate the PSD changes with frequency. A number of 5-day calculation results constitute the time-frequency color drawings as shown in Fig. 2. Figure 2a shows the geoelectric field PSD calculated by the hourly average values of 10-s sampling observational data of SHN station from January to May 2008. By compared the PSD changes before 16 March with those from 17 March till the occurrence of the Wenchuan EQ in the figure, the PSD in the latter time interval is significantly increased while obviously decreased immediately after the great EQ. From the overall changes, before the occurrence of the Wenchuan EQ, the geoelectric field PSD of $\mathrm{N}_{1} \mathrm{~S}_{1}$ channel of station SHN had an increase of 1 to 3 orders of magnitude while it decreased when approaching the date of occurrence of the great EQ. In particular, from March 16 to 20 (local time) transient and significant increase of the $T<3 h$ electric field PSD occurred, which was corresponding to the occurrence time of the 
Ms 7.3 Yutian EQ. Figure 2b shows the PSD changes of the geoelectric field calculated by the hourly average values of 10 -ssampling data of station TIA from March to June 2008. It can be seen that from 15 April to the occurrence of the Wenchuan EQ, there was a significant PSD increase in $\mathrm{N}_{1} \mathrm{~S}_{1}$ channel of station TIA, larger than the PSD at any time, which reached the maximum and then gradually decreased, returning to the value level before 15 April, immediately after the Wenchuan EQ. Figure 2c shows the PSD changes calculated by the hourly average values of minute-sampling data of station CDU from January to July 2008. Before the Wenchuan EQ, the PSD of the electric field in the EW channel of CDU increased overtly, which showed the following aspects:

(i) From 16 January to the occurrence of the EQ (nearly 4 months), the PSD of each low-frequency had fluctuating changes, while they were relatively stable after the EQ. This may be caused by the great affects from the focal preparation of the EQ due to the small distance between the station and the epicenter.

(ii) Roughly from 10 April, the PSD of low-frequency geoelectric field (particularly period $T<3 h$ components) obviously increased as compared to those in the past 40 days, with the maximum increase reaching 3 orders of magnitude; then since 30 April (12 days before the great EQ), the PSD decreased to the minimum.

(iii) Around the occurrence time of the Yutian Ms 7.3 EQ (local time, 21-25 March), the PSD alternately increased and reduced, which showed a remote relevance between the PSD changes and the epicenter of the Ms 7.3 EQ.

It can be seen from the comparison between Figs. 2 and 1 that:

(i) Roughly from 15 January to 1 March, before the Ms 8.0 Wenchuan EQ, CDU station that was nearest to the epicenter had a prominent PSD increase of the near-DC component geoelectric field; and during this period of time, the three components of ionospheric electric field also had the PSD significant increase with the $0 \sim$ a few $\mathrm{Hz}$ frequency, both of which corresponded in time.

(ii) On the eve of the Yutian Ms 7.3 EQ, in the period of geoelectric field PSD increase of SHN station (calculated by the data of 16 to 20 March, local time), the ionospheric electric field $Y$-component had an obvious PSD increase of $0 \sim$ a few $\mathrm{Hz}$ on 20 March, basically consistent with the ground electric field PSD increase in time.

(iii) During the period from 30 April to the occurrence of the Wenchuan EQ, the $X$ - and $Y$-components of the ionospheric electric field had the PSD increase with $0 \sim$ a few $\mathrm{Hz}$ frequency, but the three ground stations had no such phenomena. 


\subsubsection{Ionospheric electric field (Demeter's revisit orbits)}

Figure 3 shows the time-frequency PSD of the ionospheric electric field of the Demeter's revisited orbits above the Wenchuan EQ. The revisited period of was 16 days before the end of June 2008 and that was about 14 days after this. The time when the revisited orbits run above Wenchuan was 14:50:30 UT, roughly in the range of $\mathrm{N} 20^{\circ}-40^{\circ}$ and E100. $8^{\circ}-105.4^{\circ}$ and with the operating altitude of $660 \mathrm{~km}$. The data of a total of 90 half-orbits in the period from 1 January 2006 to 31 December 2009 are selected. Within 90 days of running 90 half orbits, only on 5 April 2006, Kp $>4$ and $|D s t|<40$. It can be seen from the figure that the significant PSD increase of electric field $(f \leq 2 \mathrm{~Hz})$ appeared above Wenchuan from May to June 2006, June to November 2007, May to September 2008, and from July to October 2009. During these periods, a series of strong EQs and great EQs happened in the Qinghai-Tibet Plateau and its surrounding areas as well as in Indonesia. Nevertheless, the PSD increase of the ionospheric electric field observed by the revisited orbits behaved complicatedly, on the whole. It is difficult to analyze the correspondence in time between the EQs and the PSD increases, and no significant anomaly appeared before the Wenchuan EQ.
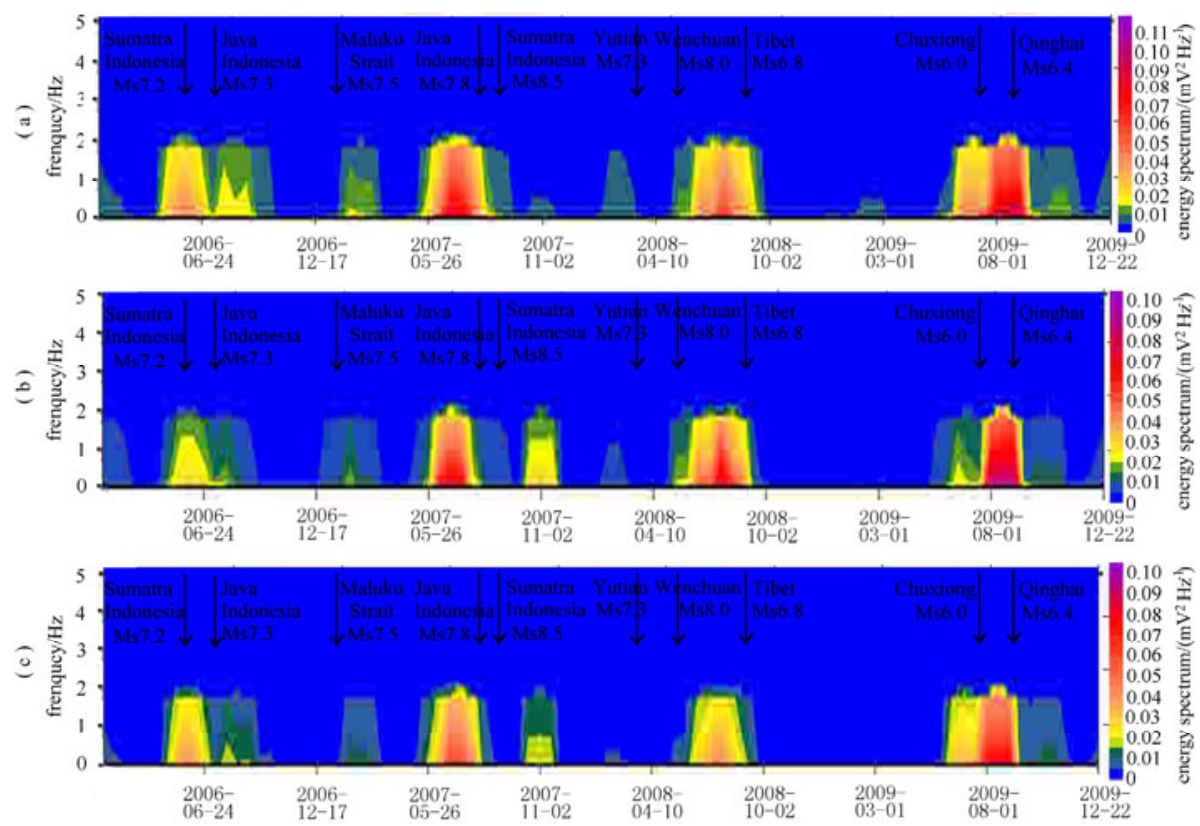

Fig. 3. PSD time-frequency diagram of electric field of the Demeter's revisit orbits above Wenchuan. Note: (a)-(c) represent the $X$-, $Y$-, and $Z$-components, respectively. 


\subsection{Ionospheric electric field changes of the Sumatra $M s 8.7$ and 8.5 EQs (continual orbit)}

On 26 December 2004, an Ms 8.7 EQ happened in the sea west of Sumatra, and its epicenter was located at N3.9 ${ }^{\circ}$ and E95.9 ${ }^{\circ}$. On 28 March 2005, an Ms 8.5 EQ occurred north of Sumatra, and its epicenter was located at N2.2 ${ }^{\circ}$ and $\mathrm{E} 97.0^{\circ}$. The distance between the two epicenters is very short. The data of the Demeter's orbits in the range of $\mathrm{S}^{\circ}-\mathrm{N} 15^{\circ}$ and $\mathrm{E} 85^{\circ}-105^{\circ}$, above Sumatra, are selected, and the data was from September 2004 to April 2005. Figure 4 shows the PSD changes with time of the ionospheric electric field, namely, the $X-, Y$-, and $Z$-components of the electric field (the EQs in the figure are marked in UT). The PSD of electric field is calculated by the MEM method. From September 2004 to April 2005, within the area below the selected the Demeter's orbits, three $M s \geq 7.0 \mathrm{EQs}$ happened, including the $M s 7.0$ EQ on 1 January $2005\left(\mathrm{~N} 5.2^{\circ}\right.$, E92.3 ${ }^{\circ}$ ), the $M s 7.0$ and 8.7 EQs which occurred in the sea west of Sumatra. In addition, an Ms 7.3 EQ happed in Sumatra $\left(\mathrm{S} 2.3^{\circ}, \mathrm{E} 104.1^{\circ}\right)$ on 25 July 2004 . In Figure 4, from early December 2004 to early February 2005, and from middle March to middle
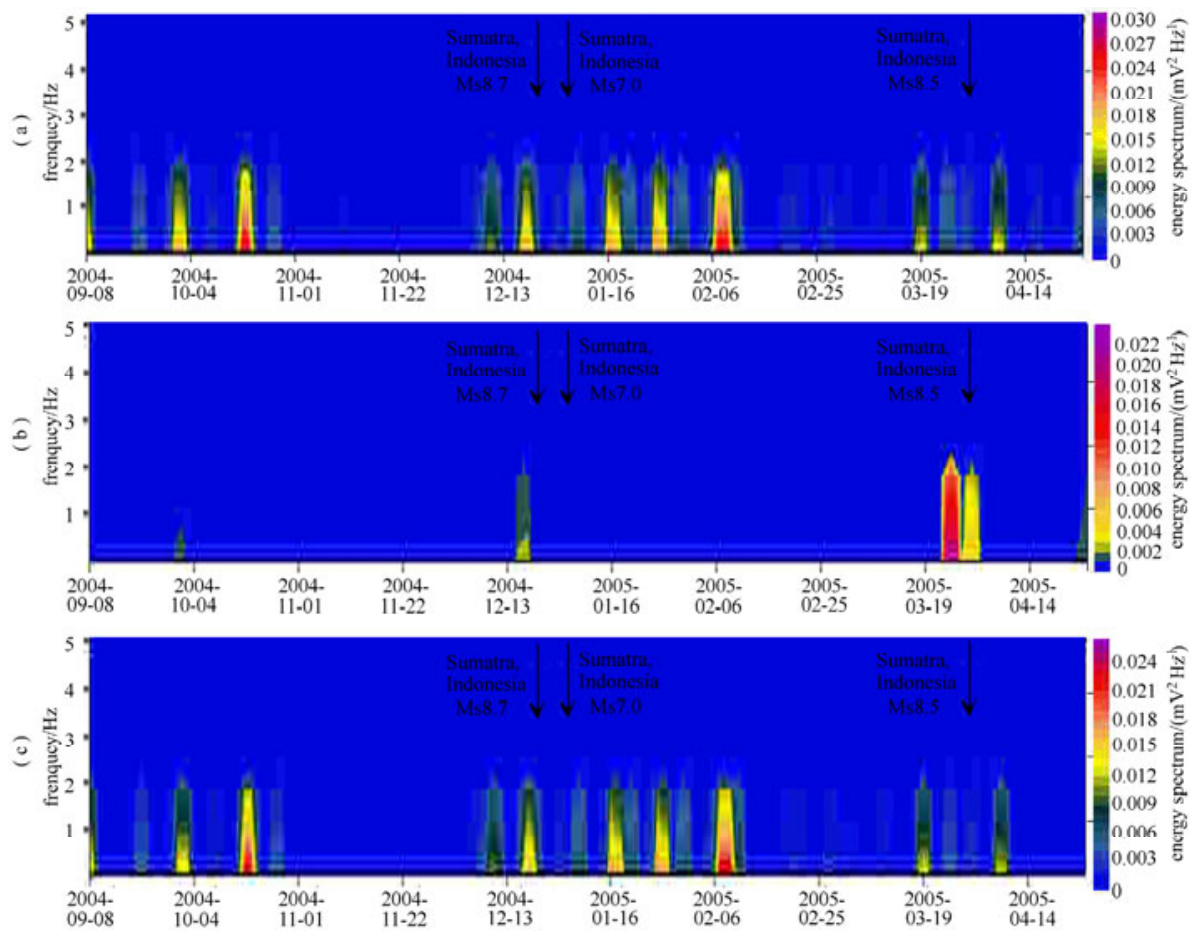

Fig. 4. PSD diagrams of the electric field of the Demeter's Up Orbit above the Sumatra EQs. Note: (a)-(c) represent the $X$-, $Y$-, and $Z$-components, respectively. 
April 2005, the PSD of the ionospheric electric field of $f<3 \mathrm{~Hz}$ increased, which was corresponding to the occurrence of the three EQs in Sumatra in time. Especially in the diagram of the $Y$-component of electric field, the increase phenomena of PSD obviously corresponded to the Ms 8.7 and 8.5 EQs. From September to October 2004, the $X$ - and Z-components of the ionospheric electric field had the PSD increase, but no EQs above $M s 7.0$ happened in the zone below the selected Demeter's orbits and its surrounding zone during the period of time before and after the PSD increase.

\subsection{Ionospheric electric field changes of the Chile Ms 8.8 EQ}

At 06:34:16 UT on 27 February 2010, an $M s$ 8.8 EQ occurred in Concepcion, Chile, with its epicenter located at $\mathrm{S} 35.8^{\circ}$, W72.7 $7^{\circ}$. Taking the epicenter of Chile EQ as the center, we selected the electric field data of Demeter's orbits in the rectangular range of $\mathrm{S} 30^{\circ}-\mathrm{S} 50^{\circ}$ and $\mathrm{W} 70^{\circ}-\mathrm{W} 90^{\circ}$, and in the period of time from 1 September 2009 to 31 March 2010. Figure 5 shows the PSD changes of the three components of the ionospheric electric field of the
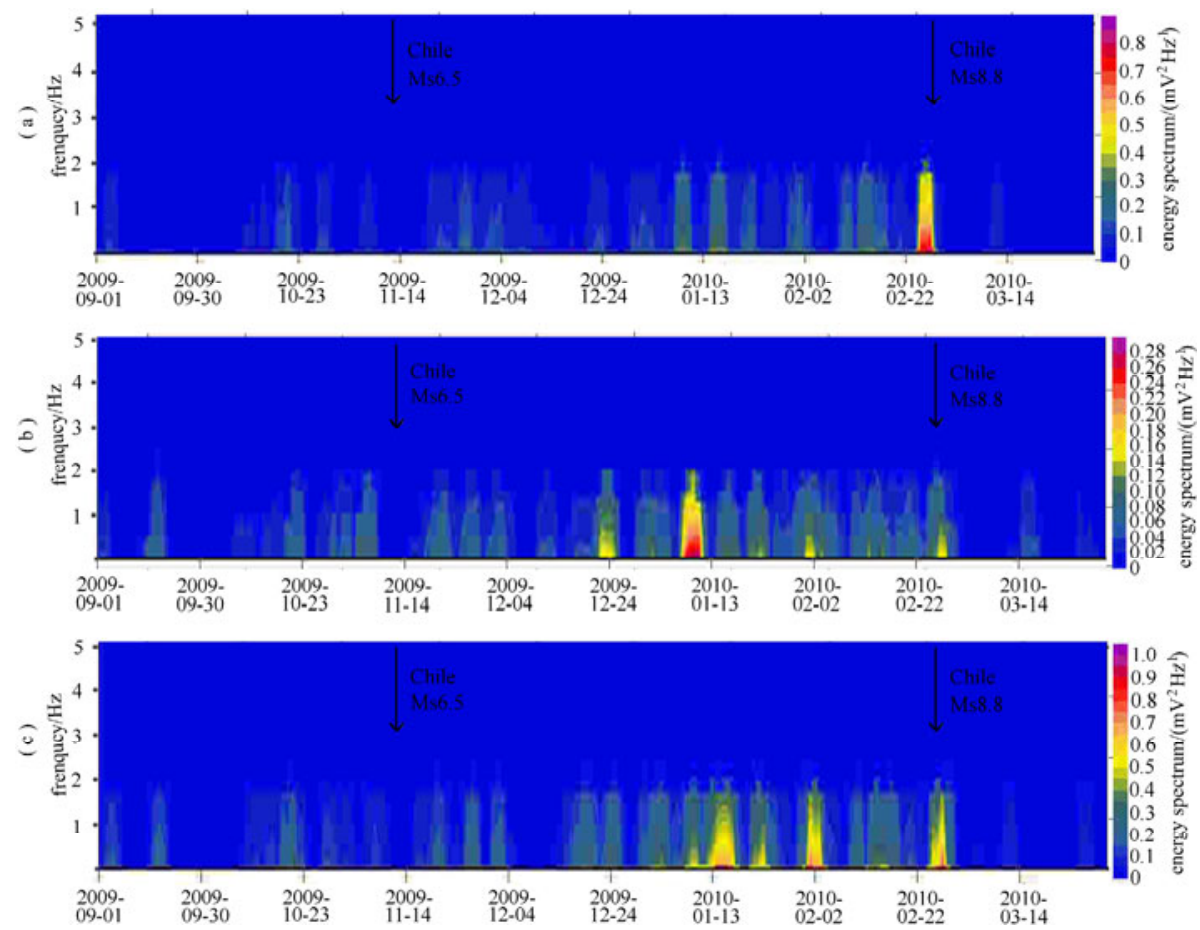

Fig. 5. PSD diagrams of the electric field of the Demeter (Up Orbits) above the Chile Ms 8.8 EQ from 1 September 2009 to 31 March 2010. Note: (a)-(c) represent the $X-, Y$-, and $Z$ - components, respectively. 
orbits above the epicenter of Chile EQ (the EQs in the figure are marked in UT). It can be seen from the figure that before the Chile $M s 8.8 \mathrm{EQ}$, the three components had significant PSD increases, which disappeared immediately after the EQ; among them, the PSD increase phenomena of $X$-component on the eve of the EQ was the most prominent. On 13 November 2009, a Ms 6.5 EQ $\left(\mathrm{S} 19.3^{\circ}, \mathrm{W} 70.3^{\circ}\right)$ occurred in the coast offshore area of northern Chile and Fig. 5 shows that there was no PSD increase before the EQ as in the case of the Ms 8.8 EQ, perhaps due to its small magnitude.

\subsection{Interim summaries}

a The PSD increase of the low-frequency components appeared, below a few $\mathrm{Hz}$ and in the ionospheric electric field observed by the Demeter when approaching the date of the Wenchuan Ms 8.0 EQ. Their frequencies were below a few $\mathrm{Hz}$ and basically consistent with the PSD increase of the ground electric field in time.

- When approaching the date of such great EQs as the Wenchuan Ms 8.0 EQ, the Yutian $M s 7.3 \mathrm{EQ}$, and the Sumatra $M s$ 8.7/8.5 EQ, especially before the Chile Ms 8.8 EQ, the PSD of the ionospheric electric field observed by the Demeter had a significant increase in low-frequency components.

- The low-frequency PSD increase of the ionospheric electric field seems to correspond to those of the geoelectric field in the time immediately before the Ms 8.0 Wenchuan and Ms 7.3 Yutian EQs and their strong aftershocks.

a The phenomena of the PSD increase of the ionospheric electric field observed by the Demeter's revisited orbits are very complicated, so it is quite difficult to analyze the correspondence in time between the EQ and the PSD increase.

\section{DISCUSSION ON REASONS OF THE GROUND AND IONOSPHERIC ELECTRIC FIELD CHANGES BEFORE EQS}

According to the literature (Du et al. 2000, Du 2011), in the late preparation stage of strong EQs (several months to one-year scale before EQs), in the focal area and nearby, the amplitude and duration of apparent resistivity (AR, for short) anomalies show a non-linear relationship with an increase of EQ magnitude as follows: for the small-magnitude EQs, the amplitude of anomaly increase slowly, whereas the duration of anomaly grows rapidly; for the large-magnitude EQs, the situation is opposite. This relationship indicates the non-linear development of the micro-cracks within the underground medium within or nearby focal areas in the late preparation stage of strong EQs by the actual EQ cases, supporting the forecast of the IPE model that the mi- 
cro-cracks are an avalanche development in the focal area (Mjachkin et al. 1975) and the inference that the micro fracture amount within the medium of focal area increases non-linearly in the late preparation stage (Kusunose et al. 1980). Du et al. (2007) has studied the AR anisotropic changes nearby epicentral areas of moderate and above EQs in the late EQ preparation stage, and came to the following conclusion: the primary reason for the anisotropic changes is due to the deformation of underground medium, which will conduct a micro-crack directional alignment or preponderant orientation effect in the process of medium deformation. From this point of view, in the later preparation stage of a strong EQ, the non-linearly and directionally aligned medium process of the micro cracks occurred really within the medium in the focal area or its vicinity. The DD model (Nur 1972, Scholz et al. 1973) emphasized the role of groundwater in the medium within a focal region, and other publications (Du et al. 2007, Du 2011) stressed the significant contribution of water to AR changes in the aforementioned medium process. These research of mechanism on AR changes before EQs reveals that in the later preparation stage of a strong EQ, two physical processes of medium may exist in a focal region and its vicinity, such as the non-linear development of the micro cracks within the medium, and their directional alignment or preponderant orientation effect. The reason for the electromagnetic radiation before an EQ could be also associated with these two physical processes. Therefore, it can be believed that in the later stage of an EQ preparation the physical processes occurred within the medium in the focal area or its vicinity, which caused the water migration due to the chaining effect of the micro cracks and made the conducting paths in the medium connected, and almost immediately induced the changes of the natural electric field due to the electronic and ionic conduction mechanism; in this process, such factors as simultaneous occurrence of "the mechanical to electrical conversion effect" and the water migration also excited the intensely lowfrequency electromagnetic radiation.

A number of scholars have studied the possible reason why the lowfrequency geo-electromagnetic signals might be propagated to the ionosphere from the underground electromagnetic radiation source (Molchanov et al. 1995, Shalimov and Gokhberg 1998, Huang 2002, Pulinets 2004, Sorokin et al. 2005). According to the calculations for the upward propagation of the electromagnetic waves by Molchanov et al. (1995), the highfrequency components in the wide-band electromagnetic waves caused by the EQ preparation are mostly absorbed when they propagated to the surface from the focal area; consequently, of the electromagnetic waves that can reach the earth surface, only electromagnetic waves with the frequency below $10-20 \mathrm{~Hz}$ can penetrate the atmosphere and be observed by the satellite. 
This paper has processed and studied the ionospheric electric field data with the sampling frequency of $39.0625 \mathrm{~Hz}$ observed by Demeter; it was found that before an impending great EQs, only the PSD increase of the lowfrequency components of electric field, a few $\mathrm{Hz}$ or even $\mathrm{DC}$, appeared prominently, consistent with the results calculated by Molchanov et al. (1995). Supposing the PSD increase of the low-frequency ionospheric electric field that is corresponding to the EQs is spread to the earth surface from the focal region, and then propagated to the ionosphere, and taking the atmospheric conductivity $\sigma=1 \times 10^{-4} \mathrm{~s} / \mathrm{m}$, relative permeability $\mu_{r}=1$ and relative dielectric constant $\varepsilon_{r}=1$, it can be estimated that the minimum propagation distance of the electric wave of $20 \mathrm{~Hz}$ to the atmosphere is $300 \mathrm{~km}$, and that of the electric wave of $2 \mathrm{~Hz}$ is around $1000 \mathrm{~km}$. The running altitude of the Demeter satellite is $710 \mathrm{~km}$ (which changes into $665 \mathrm{~km}$ in mid-December 2005), and the PSD increase of a few Hz components of the ionospheric electric field are the most prominent before the Wenchuan EQ, the Sumatra EQ, and the Chile EQ. Pulinets and Boyarchuk (2004) have discussed how the anomalous electric field generated in the seismogemnic area penetrates through the ionosphere and prompts the non-uniform plasma in the ionosphere, so it can be believed that the phenomena of the PSD increase corresponding to the EQs may be caused by the propagation of lowfrequency electromagnetic wave from the ground to the ionosphere.

\section{CONCLUSIONS}

a The phenomena of the PSD increase of the low-frequency components (DC to a few $\mathrm{Hz}$ ) in the ionospheric electric field observed by Demeter satellite appeared before the Ms 8.0 Wenchuan EQ and strong aftershocks, the Ms 7.3 Yutian EQ, the Ms 8.7/8.5 Sumatra EQs, and the Ms 8.8 Chile EQ; these were mainly the imminent anomalies, more significant for the short-distance and large-magnitude EQs.

a Before the occurrence of the Ms 8.0 Wenchuan EQ and the Ms 7.3 Yutian $\mathrm{EQ}$, the PSD increase of near-DC components in the geoelectric field appears also in several ground electromagnetic stations in the northeastern and western edges of the Qinghai-Tibet Plateau, and agrees in time with the PSD increase of the low-frequency components in the ionospheric electric field. This shows a good correspondence between the ionospheric and ground electromagnetic phenomena before the two great EQs. In particular, when approaching the date of occurrence of the Ms 7.3 Yutian $\mathrm{EQ}$, the SHN station recorded the significant PSD increase in the geoelectric field during the period of the PSD increase of the $Y$ component in the ionospheric electric field. 
- It is rather difficult to analyze the PSD anomalies in the ionospheric electric field before EQs due to their complicated time, spatial, and intense distributions. There sometimes existed the confusing phenomena. For example, the prominent anomaly appeared before the long-distance great EQs while no anomaly before the short-distance EQs, and a smallamplitude anomaly appeared before the large-magnitude EQs or even no anomaly, while the prominent anomaly appeared before the smallmagnitude EQs. This is similar to the results observed by the ground stations. Hence, it is very difficult to discern the earthquake-related anomaly in the ionospheric electric field observed by the Demeter's revisited orbits.

Acknowledgment. This work was supported by the Basic Scientific Research Special Fund of Institute of Earthquake Prediction (2012IESLZ06) and Natural Science Foundation of China (41374080). Thanks are extended to Demeter website (http://demeter.cnrs-orleans.fr/) for providing the ionospheric magnetic field data, and thanks are given to Professor Jacques Zlotnicki at France National Center for Scientific Research and Professor Mechel Parrot at the Orleans University in France.

\section{References}

Adler, P.M., J.L. Le Mouël, and J. Zlotnicki (1999), Electrokinetic and magnetic fields generated by flow through a fractured zone: A sensitivity study for La Fournaise volcano, Geophys. Res. Lett. 26, 6, 795-798, DOI: 10.1029/ 1999GL900095.

Berthelier, J.J., M. Godefroy, F. Leblanc, M. Malingre, M. Menvielle, D. Lagoutte, J.Y. Brochot, F. Colin, F. Elie, C. Legendre, P. Zamora, D. Benoist, Y. Chapuis, J. Artru, and R. Pfaff (2006), ICE, the electric field experiment on DEMETER, Planet. Space Sci. 54, 5, 456-471, DOI: 10.1016/j.pss.2005. 10.016 .

Cress, G.O., B.T. Brady, and G.A. Rowell (1987), Sources of electromagnetic radiation from fracture of rock samples in the laboratory, Geophys. Res. Lett. 14, 4, 331-334, DOI: 10.1029/GL014i004p00331.

Cussac, T., M.A. Clair, P. Ultré-Guerard, F. Buisson, G. Lassalle-Balier, M. Ledu, C. Elisabelar, X. Passot, and N. Rey (2006), The Demeter microsatellite and ground segment, Planet Space Sci. 54, 5, 413-427, DOI: 10.1016/j.pss. 2005.10.013.

Du, X.B. (2011), Two types of changes in apparent resistivity in earthquake prediction, Sci. China D 54, 1, 145-156, DOI: 10.1007/s11430-010-4031-y. 
Du, X.B., H.Y. Zhao, and B.Z. Chen (1993), On the relation of imminent sudden change in earth resistivity to the active fault and earthquake generating stress field, Acta Seismol. Sin. 6, 3, 663-673, DOI: 10.1007/BF02650405.

Du, X.B., Y.W. Liu, and M.K. Ni (1997), On the spatial characteristic of the shortterm and imminent anomalies of underground water behaviors before strong earthquake, Acta Seismol. Sin. 10, 4, 523-533, DOI: 10.1007/ s1 1589-997-0062-7.

Du, X.B., S.Z. Xue, Z. Hao, and S.Z. Zhang (2000), On the relation of moderateshort term anomaly of earth resistivity to earthquake, Acta Seismol. Sin. 13, 4, 393-403, DOI: 10.1007/s11589-000-0021-z.

Du, X.B., J.L. Xi, D.C. Tan, J.L. Zhao, J.D. Qian, Y.Q. Lu, J. Lu, Y.X. Tang, H.L. Kang, Y.F. Chen, and D.Z. Wang (2006), Specification for the construction of seismic station geoelectrical station. Part 2: Geoelectrical field observatory, DB/T 18.2-2006, The Seismology Trades Standard of the PRC, 2-5 (in Chinese).

Du, X.B., N. Li, Q. Ye, Z.H. Ma, and R. Yan (2007), A possible reason for the anisotropic changes in apparent resistivity near the focal region of strong earthquake, Chinese J. Geophys. 50, 6, 1555-1565, DOI: 10.1002/cjg2.1175 (in Chinese).

Enomoto, Y., and H. Hashimoto (1992), Transient electrical activity accompanying rock under indentation loading, Tectonophysics 211, 1-4, 337-344, DOI: 10.1016/0040-1951(92)90069-1.

Enomoto, Y., H. Hashimoto, N. Shirai, Y. Murakami, T. Mogi, M. Takada, and M. Kasahara (2006), Anomalous geoelectric signals possibly related to the 2000 Mt. Usu eruption and 2003 Tokachi-Oki earthquakes, Phys. Chem. Earth 31, 4-9, 319-324, DOI: 10.1016/j.pce.2006.02.013.

Fan, Y.Y., X.B. Du, J. Zlotnicki, D.C. Tan, Z.H. An, J.Y. Chen, G.L. Zheng, J. Liu, and T. Xie (2010), The electromagnetic phenomena before the $\mathrm{M}_{\mathrm{s}} 8.0$ Wenchuan earthquake, Chinese J. Geophys. 53, 6, 997-1010, DOI: 10.1002/ cjg2.1570.

He, Y.F., D.M. Yang, H.R. Chen, J.D. Qian, R. Zhu, and M. Parrot (2009), SNR changes of VLF radio signals detected onboard the DEMETER satellite and their possible relationship to the Wenchuan earthquake, Sci. China D 52, 6, 754-763, DOI: 10.1007/s11430-009-0064-5.

Huang, Q.H. (2002), One possible generation mechanism of co-seismic electric signals, Proc. Japan Acad. B 78, 7, 173-178, DOI: 10.2183/pjab.78.173.

Huang, Q.H. (2005), Controlled analogue experiments on propagation of seismic electromagnetic signals, Chin. Sci. Bull. 50, 17, 1957-1961, DOI: 10.1360/ 982004-312.

Huang, Q.H., and M. Ikeya (1998), Seismic electromagnetic signals (SEMS) explained by a simulation experiment using electromagnetic waves, Phys. Earth Planet. In. 109, 3-4, 107-114, DOI: 10.1016/S0031-9201(98)00135-6. 
Ishido, T., and J.W. Pritchett (1999), Numerical simulation of electrokinetic potentials associated with subsurface fluid flow, J. Geophys. Res. 104, B7, 15247-15259, DOI: 10.1029/1999JB900093.

Kusunose, K., I.K. Yamamoto, and T. Hirasawa (1980), Source process of microfracture in granite with reference to earthquake prediction, Sci. Rep. Tohoku Univ. Geophys. 26, 3-4, 111-121.

Liu, J.Y., Y.I. Chen, Y.J. Chuo, and S.A. Pulinets (2003), A statistical study of ionospheric precursors of $\mathrm{M}>5.0$. In: Proc. EGS-AGU-EUG Joint Assembly, 6-11 April 2003, Nice, France, Abstr. no. 4780.

Liu, J., X.B. Du, J. Zlotnicki, Y.Y. Fan, Z.H. An, T. Xie, G.L. Zheng, D.C. Tan, and J.Y. Chen (2011), The changes of the ground and ionosphere electric/ magnetic fields before several great earthquakes, Chinese J. Geophys. 54, 11, 2885-2897, DOI: 10.3969/j.issn.0001-5733.2011.11.018.

Ma, Q.Z., Z.S. Feng, Z.P. Song, and W.G. Zhao (2004), Study on the variation characteristics of the geoelectric field preceding earthquakes, Acta Seismol. Sin. 17, 3, 334-343, DOI: 10.1007/s11589-004-0055-8.

Michel, S., and J. Zlotnicki (1998), Self-potential and magnetic surveying of La Fournaise volcano (Réunion Island): Correlations with faulting, fluid circulation, and eruption, J. Geophys. Res. 103, B8, 17845-17857, DOI: 10.1029/98JB00607.

Mjachkin, V.I., W.F. Brace, G.A. Sobolev, and J.H. Dieterich (1975), Two models for earthquake forerunners, Pure Appl Geophys. 113, 1, 169-181, DOI: 10.1007/BF01592908.

Molchanov, O.A., M. Haykawa, and V.A. Rafalsky (1995), Penetration characteristics of electromagnetic emissions from an underground seismic source into the atmosphere, ionosphere, and magnetosphere, J.Geophys. Res. 100, A2, 1691-1712, DOI: 10.1029/94JA02524.

Nagamoto, H., T. Fukushima, Y. Ida, Y. Matsudo, and M. Hayakawa (2008), Disturbances in VHF/UHF telemetry links as a possible effect of the 2003 Hokkaido Tokachi-oki earthquake, Nat. Hazards Earth Syst. Sci. 8, 4, 813817, DOI: 10.5194/nhess-8-813-2008.

Nur, A. (1972), Dilatancy, pore fluids, and premonitory variations of $t_{s} / t_{p}$ travel times, Bull. Seismol. Soc. Am. 62, 5, 1217-1222.

Ogawa, T., K. Oike, and T. Miura (1985), Electromagnetic radiations from rocks, J. Geophys. Res. 90, D4, 6245-6249, DOI: 10.1029/JD090iD04p06245.

Parrot, M. (1995), Use of satellites to detect seismo-electromagnetic effects, $A d v$. Space Res. 15, 11, 27-35, DOI: 10.1016/0273-1177(95)00072-M.

Parrot, M. (ed.) (2006a), First Results of the DEMETER Micro-satellite, Planet. Space Sci. 54, 5.

Parrot, M. (2006b), Special issue of Planetary and Space Science'DEMETER', Planet. Space Sci. 54, 5, 411-412, DOI: 10.1016/j.pss.2005.10.012. 
Pulinets, S. (2004), Ionospheric precursors of earthquakes; Recent advances in theory and practical applications, Terr Atmos. Ocean Sci. 15, 3, 413-435.

Pulinets, S., and K. Boyarchuk (2004), Ionoshperic Precursors of Earthquakes, Springer, Berlin, 315 pp.

Pulinets, S.A., A. Leyva Contreras, G. Bisiacchi-Giraldi, and L. Ciraolo (2005), Total electron content variations in the ionosphere before the Colima, Mexico, earthquake of 21 January 2003, Geophys. Int. 44, 4, 369-377.

Qian, J.D., Z.W. Guo, J.L. Zhao, D.M. Yang, J.L. Xi, Y.F. Gao, J.P. Zhou, X.J. Mao, Z.B. Zheng, G.Z. Zhao, X. Zhou, S.L. Ma, X.B. Chen, J.J. Wang, Q.Z. Ma, D.C. Tan, Y.X. Tang, and T.Q. Yang (2004), Technical requirement for observational environment of seismic stations - Part 2: Electromagnetic observation, GB/T 19531.2-2004, The Standard of the PRC, 1429 (in Chinese).

Scholz, C.H., L.R. Sykes, and Y.P. Aggrawal (1973), Earthquake prediction: A physical basis, Science 181, 4102, 803-810, DOI: 10.1126/science.181. 4102.803.

Shalimov, S., and M. Gokhberg (1998), Lithosphere-ionosphere coupling mechanism and its application to the earthquake in Iran on June 20, 1990. A review of ionospheric measurements and basic assumptions, Phys. Earth Planet. In. 105, 3-4, 211-218, DOI: 10.1016/S0031-9201(97)00092-7.

Sorokin, V.M., V.M. Chmyrev, and A.K. Yaschenko (2005), Theoretical model of DC electric field formation in the ionosphere stimulated by seismic activity, J. Atmos. Sol.-Terr. Phys. 67, 14, 1259-1268, DOI: 10.1016/j.jastp.2005. 07.013.

Tang, J., Y. Zhan, L.F. Wang, J.L. Xu, G.Z. Zhao, X.B. Chen, Z.Y. Dong, Q.B. Xiao, J.J. Wang, J.T. Cai, and G.J. Xu (2008), Coseismic signal associated with aftershock of the Ms8.0 Wenchuan earthquake, Seismol. Geol. 30, 3, 739-745, DOI: 10.3969/j.issn.0253-4967.2008.03.012.

Telesca, L., and K. Hattori (2007), Non-uniform scaling behavior in ultra-lowfrequency (ULF) earthquake-related geomagnetic signals, Physica A 384, 2 , 522-428, DOI: 10.1016/j.physa.2007.05.040.

Telesca, L., G. Colangelo, and V. Lapenna (2005), Multifractal variability in geoelectrical signals and correlations with seismicity: a study case in southern Italy, Nat. Hazards Earth Syst. Sci. 5, 5, 673-677, DOI: 10.5194/nhess5-673-2005.

Telesca, L., V. Lapenna, M. Macchiato, and K. Hattori (2008), Investigating nonuniform scaling behavior in Ultra Low Frequency (ULF) earthquake-related geomagnetic signals, Earth Planet. Sci. Lett. 268, 1-2, 219-224, DOI: 10.1016/j.eps1.2008.01.033.

Telesca, L., M. Lovallo, A. Ramirez-Rojas, and F. Angulo-Brown (2009), A nonlinear strategy to reveal seismic precursory signatures in earthquake-related self-potential signals, Physica A 388, 10, 2036-2040, DOI: 10.1016/j.physa. 2009.01.035. 
Uyeda, S., M. Hayakawa, T. Nagao, O. Molchanov, K. Hattori, Y. Orihara, K. Gotoh, Y. Akinaga, and H. Tanaka (2002), Electric and magnetic phenomena observed before the volcano-seismic activity in 2000 in the Izu Island Region, Japan, Proc. Natl. Acad. Sci. USA 99, 11, 7352-7355, DOI: 10.1073/ pnas.072208499.

Varotsos, P., and M. Lazaridou (1991), Latest aspects of earthquake prediction in Greece based on seismic electric signals, Tectonophysics 188, 3-4, 321-347, DOI: 10.1016/0040-1951(91)90462-2.

Varotsos, P., K. Alexopoulos, K. Nomicos, and M. Lazaridou (1986), Earthquake prediction and electric signals, Nature 322, 6075, 120, DOI: 10.1038/ $322120 \mathrm{a} 0$.

Varotsos, P., N. Sarlis, M. Lazaridou, and P. Kapiris (1998), Transmission of stress induced electric signals in dielectric media, J. Appl. Phys. 83, 1, 60-70, DOI: $10.1063 / 1.366702$.

Yamada, I., K. Masuda, and H. Mizutani (1989), Electromagnetic and acoustic emission associated with rock fracture, Phys. Earth Planet. In. 57, 1-2, $157-$ 168, DOI: 10.1016/0031-9201(89)90225-2.

Zhang, X.M., H.R. Chen, J. Liu, X.H. Shen, Y.Q. Miao, X.B. Du, and J.D. Qian (2012), Ground-based and satellite DC-ULF electric field anomalies around Wenchuan M8.0 earthquake, Adv. Space Res. 50, 1, 85-95, DOI: 10.1016/ j.asr.2012.03.018.

Zlotnicki, J., J.L. Le Mouël, Y. Sasai, P. Yvetot, and M.H. Ardisson (2001), Selfpotential changes associated with volcanic activity: Short-term signals associated with March 9, 1998 eruption on La Fournaise volcano (Réunion Island), Ann. Geophys. 44, 2, 335-354, DOI: 10.4401/ag-3600.

Received 24 December 2013 Received in revised form 24 June 2014 Accepted 4 July 2014 